\title{
Prolonging Network Lifetime via Partially Controlled Node Deployment and Adaptive Data Propagation in WSN
}

\author{
Fangting Sun, Mark Shayman \\ Department of Electrical and Computer Engineering \\ University of Maryland, College Park, MD 20742 \\ Email: \{ftsun, shayman\}@eng.umd.edu
}

\begin{abstract}
In large scale wireless sensor networks, maximizing the network lifetime under energy constraint is one of the most critical tasks. In this paper we investigate how to maximize the wireless sensor network lifetime via joint relay deployment and adaptive data propagation. We consider wireless sensor networks with two types of nodes: sensors and relays. Sensor nodes will be deployed randomly under certain coverage constraint, and relay nodes will be deployed in a partially controlled way such that the network lifetime can be maximally extended. We formulate the joint relay deployment and adaptive data propagation problem into a mixed-integer nonlinear programming (MINLP) problem. Since MINLP is NPhard in general, in this paper we have proposed a greedy heuristic to attack this problem. The numerical results have demonstrated that significant network lifetime extension can be achieved if relay nodes can be deployed in an effective way. For example, when the proposed joint scheme is used, adding $10 \%$ extra relay nodes can extend the network lifetime by $50 \%$ further comparing to using adaptive data propagation solely for a large scale sensor network with 2000 nodes. We then conducted a set of simulations to verify the numerical results. Since some approximation has been made when solving the problem numerically, lifetime extension obtained by the numerical solution is slightly higher than the network lifetime extension obtained in the simulation. However, the network lifetime extension is still significant.
\end{abstract}

\section{INTRODUCTION}

During the last decade, wireless sensor networks have drawn extensive attention due to the wide range

Research partially supported by AFOSR under grant F496200210217 and NSF under grant CNS-0519554. of promising applications, such as environmental monitoring, industrial sensing and diagnostics, battlefield surveillance, target tracking, search and rescue, and disaster relief [1], [2]. To make the deployment of large scale wireless sensor networks affordable and viable, sensor nodes are usually made small and cheap, and with only limited battery supply. As a result, energy becomes one of the most precious resource in wireless sensor networks.

In a sensor network where nodes need to send data to the sink and all nodes use the same transmission power, nodes around the sink will experience much higher power consumption than faraway nodes because of the extra relaying burden. As a consequence, these nodes will run out of energy very quickly, resulting in the death of the network, though there is still considerable unused energy left in those nodes far away from the sink. This makes those nodes around the sink the major bottleneck in wireless sensor networks when trying to extend the network lifetime.

To relieve this phenomena, Luo et al. proposed to move the sink around to collect data from sensors in order to balance the energy consumption in different sensor nodes [3]. In this way, the set of sensor nodes around the sink changes over time, then the energy depletion rate between different nodes becomes similar. However, it requires complicated sink mobility pattern and complex routing algorithm for sensors to send data to the sink.

Another straightforward way is to allocate more resources into those areas with high energy consumption rate, as demonstrated in [4]-[6]. Strategic placement of sensors in linear networks was studied in [4]. [5] investigated energy provisioning problem in two-tier sensor 
networks. [6] studied the sensor deployment problem in circular sensor networks, however, they only consider the case where processing and receiving energy consumption is negligible.

In our previous work [7], we have proposed to adaptively split traffic and adjust transmission power in order to eliminate this bottleneck. We modeled the problem as a linear programming problem and the results show that significant improvement can be achieved when the proposed adaptive data propagation algorithm is used.

In this paper we focus on the situations in which there are two types of nodes in a wireless sensor network: sensor nodes and relay nodes. Sensor nodes are randomly deployed with certain network coverage requirement, and relay nodes will be deployed in a partially controlled way to further extend the network lifetime. In this work we investigate how to maximize the network lifetime via joint optimization of node deployment and adaptive data propagation. This optimization problem is mixed integer nonlinear programming problem, which is NPhard. In this work we propose a greedy algorithm to attack it. The numerical results show that significant gain can be achieved, which has also been confirmed by the simulation study.

The rest of the paper is organized as follows. Section II describes the network model and formulates the problem. Section III presents the numerical results for the joint optimization problem. Section IV provides the simulation studies. Finally Section V concludes this paper.

\section{Network Model And Problem Formulation}

In this paper we consider the following flat circular wireless sensor network model. There are two types of wireless nodes in the network: sensors and relays. Each sensor needs to submit the collected information to the sink which is located in the center of the network. Relay nodes will not generate traffic, that is, relay nodes will only forward packets, but will not generate traffic. The sensor nodes are randomly deployed according to the uniform distribution with fixed density such that the network coverage constraint can be satisfied with high probability [8], [9]. Let $N$ denote the total number of sensor nodes, which can be easily calculated. We consider the problem of deploying $M$ relay nodes into the network in a partially controlled way to prolong the network lifetime. Here partially controlled way means that the relays cannot be deployed to specific positions controllably, but can be deployed with specific distribution density. Our objective is to find the optimal relay distribution achieving maximum network lifetime when sensor nodes have a fixed and nonrenewable battery.

We assume that all nodes (sensors and relays) have the same amount of initial energy, denoted by $E$. There is no energy constraint on the sink. Given the network to be deployed, some Quality of Service (QoS) requirements, and specific types of sensors, we also pose a minimum transmission range limitation for each sensor, denoted by $r_{\min }$. The value of $r_{\min }$ can be determined by both hardware limitation and QoS requirements, such as network connectivity. We assume all nodes can adjust their transmission range as needed.

In this paper, similar as in [5], [10], we model the energy consumption at each node as follows:

$$
\begin{aligned}
P_{t}(r) & =\gamma_{1}+\beta \cdot r^{\alpha} \text { per bit } \\
P_{r} & =\gamma_{2} \text { per bit }
\end{aligned}
$$

Here $P_{t}(r)$ is the processing and transmission energy consumption and $P_{r}$ is the receiving energy consumption. $\gamma_{1}$ and $\gamma_{2}$ are constant which are determined by the underlying technology. $\alpha$ is the path loss exponent, which is determined by the environment. $r$ is the targeted transmission range, and $\beta$ is a scalar indicating the energy needed to successfully convey an information bit to a unit distance.

We assume that all nodes (sensors, relays and the sink) use a common frequency channel. We adopt the network lifetime definition presented in [11], which is the time span from the network deployment to the first loss of coverage.

To make the problem tractable, in this paper we follow the network model used in [7]. Specifically, the network is divided into multiple layers: a node belongs to the $l^{\text {th }}$ layer if and only if its distance to the sink lies in the range $\left((l-1) \cdot r_{\min }, l \cdot r_{\min }\right]$, and the layer 0 is the sink. So the width for each layer is $r_{\text {min }}$.

Due to the symmetry of network topology and traffic pattern relative to the sink, intuitively relay nodes should be deployed in a symmetric way. When the network size is large and the number of relay nodes is not too small, deploying nodes in a certain layer can be approximated by uniformly distributing extra energy to sensor nodes in this layer. The effect of this simplifying assumption will be examined through simulation. Now the original problem can be transformed to determining how to distribute the extra energy in the most efficient way such that the network lifetime can be maximized. Let $M$ denote the total number of available relay nodes, 
$N_{l}$ denote the number of sensor nodes in the $l^{\text {th }}$ layer, and $e_{l}$ is the extra energy assigned to each node in the $l^{\text {th }}$ layer.

Let $R$ denote the radius of the network and let $L$ denote the total number of layers in the network. For any integers $l, k$ with $0 \leq k<l \leq L$, let $x_{l, k}$ denote the average number of bits that a node in the $l^{\text {th }}$ layer needs to request nodes in the $k^{\text {th }}$ layer to forward per unit time. Let $g$ denote the average number of bits each sensor node will generate per unit time. We can readily check that the ratio between the number of nodes in the $k^{\text {th }}$ layer and the number of nodes in the $l^{\text {th }}$ layer $(k>l)$ is $\frac{2 k-1}{2 l-1}$. Thus the average number of bits that a node in the $l^{\text {th }}$ layer will receive from nodes in the $k^{\text {th }}$ layer $(k>l)$ should be $\frac{2 k-1}{2 l-1} x_{k, l}$. Let $T_{\text {life }}$ denote the network lifetime. Then the original problem can be simplified as follows:

$$
\begin{gathered}
\max _{\left\{x_{i, j}, e_{l}\right\}} T_{l i f e} \text { s.t. } \\
\sum_{k=l+1}^{L} \frac{2 k-1}{2 l-1} x_{k, l}+g=\sum_{k=0}^{l-1} x_{l, k}, \quad 1 \leq l \leq L \\
\sum_{k=0}^{l-1} x_{l, k} P_{t}(l, k)+\sum_{k=l+1}^{L} x_{k, l} P_{r} \leq \frac{E+e_{l}}{T_{l i f e}}, \quad 1 \leq l \leq L \\
x_{l, k} \geq 0, \quad 0 \leq k<l, \quad 1 \leq l \leq L \\
\sum_{l=1}^{L} e_{l} N_{l} \leq M \cdot E, \quad 1 \leq l \leq L \\
e_{l}=\frac{k \cdot E}{N_{l}}, \quad k \text { is a non-negative integer, } \quad 1 \leq l \leq L
\end{gathered}
$$

pointing out that once a new relay is added, the proposed adaptive data propagation scheme in [7] will be applied (5) parameters [12], [13]. Specifically, we set $\gamma_{1}=45 \mathrm{~nJ} / \mathrm{bit}$,

(6) parameter setting, there exists an optimal transmission (7) meter can be minimized, which is referred to as the (8)

where $P_{t}(l, k)=P_{t}\left((l-k) r_{\min }\right)$. Eqn. (4) models the traffic conservation, i.e., for each node the amount of transmitted traffic should be equal to the traffic received plus the traffic generated. Eqn. (5) poses the energy constraint. Eqn. (6) guarantees the feasibility of the solutions and prevents nodes sending traffic further away from the sink. Eqn. (7) applies relay nodes quantity constraint. Eqn. (8) guarantees the integrity of the relay nodes, that is, the energy of one relay node cannot be split into two parts.

\section{NUMERICAL RESUlTS}

This section is devoted to solving the optimization problem defined in Section II numerically. Since the optimization problem (3)-(8) is a mixed-integer nonlinear programming problem, which is known to be a to re-calculate the lifetime extension.

Now let us analyze the numerical solutions obtained by the proposed heuristic. To make the results solid, our analysis is based on some typical energy consumption $\gamma_{2}=135 \mathrm{~nJ} / \mathrm{bit}$, and $\beta=10 \mathrm{pJ} / \mathrm{bit} / \mathrm{m}^{2}$ for $\alpha=2$. For each range such that the energy consumption per bit per characteristic distance [11]. In our model, it is easy to check that the characteristic distance $d_{\text {char }}$ is:

$$
d_{\text {char }}=\left(\frac{\gamma_{1}+\gamma_{2}}{\beta(\alpha-1)}\right)^{\frac{1}{\alpha}} \text {. }
$$

NP-hard problem, in this paper we resort to developing Peient heuristics to solve this problem.

We attack this problem using the following greedy ployed, relay nodes will be added to the network one by one. Each time when a relay node is added to the network, it will be put in a location that can maximally extend the network lifetime, or more specifically, it will be put in a layer that leads to longest network lifetime. This is motivated by the following observation: due to the asymmetric role of each layer, some layers have a large portion of their traffic using a very long range, way by transmitting most of their traffic with a short range. This indicates that those nodes utilizing energy highly efficiently play a bottleneck role. Here it is worth

We fix $r_{\min }=d_{c h a r}$, which is the layer width. The sensor node density is set to be $\frac{20}{\pi \times d_{\text {char }}^{2}}$, which guarantees the network is 3-connected with probability more than $99.99 \%$ [14]. Here we further assume the sensor sense area radius is $d_{\text {char }}$. So the sensor density will guarantee the sensing coverage too.

To demonstrate the network lifetime that can be further extended by applying joint relay deployment and adaptive data propagation, we regard the proposed adaptive data propagation scheme, which allows each sensor send traffic to multiple destinations in multiple layers to maximize the network lifetime [7], as the baseline, and normalize the network lifetime achieved by this baseline scheme as 1 . Besides the above proposed greedy heuristics, we have also studied the gain achieved by randomly deploying those relay nodes in the network. Specifically, in this case the extra energy provided by the 


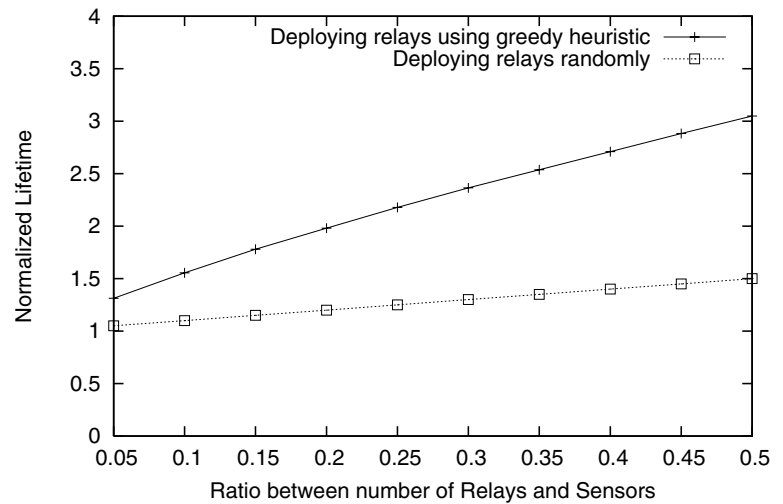

(a)

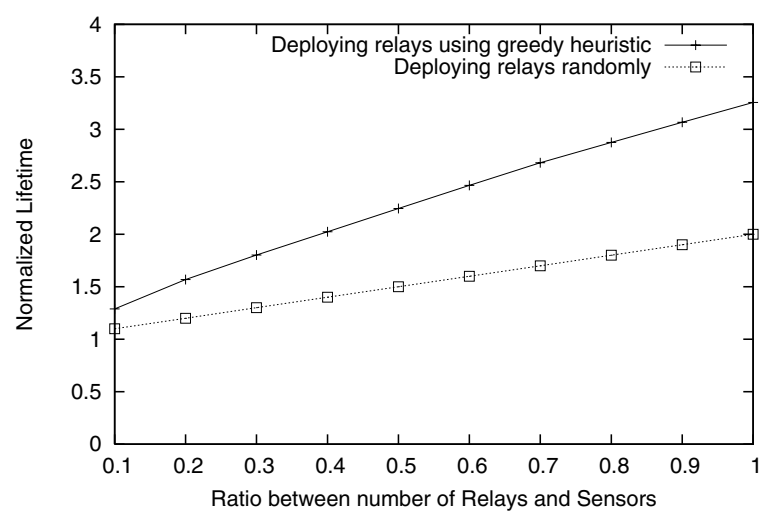

(b)

Fig. 1. Normalized network lifetime by deploying extra relays (a) 10-layer network (b) 5-layer network

relay nodes will be distributed uniformly to the sensor nodes.

Fig. 1 illustrates the numerical results for different network size with different number of extra relay nodes. Specifically, $\mathrm{X}$-axis denotes the total number of relays that will be put to the network, and Y-axis denotes the normalized network lifetime. The two curves represent the achieved network lifetime by applying two different relay deployment scheme: one is solving the optimization problem (3)-(8) by the above greedy heuristic and one is deploying the relays randomly. In both schemes, the adaptive data propagation scheme [7] has been applied.

Fig. 1(a) shows the results for the network with radius $10 d_{\text {char. }}$. That is, there are 10 layers in the network. Based on the network size and sensor node density, it is easy to calculate that there are 2000 sensors in the network. From these results we can see that if we can deploy relays in an effective way, significant gain can

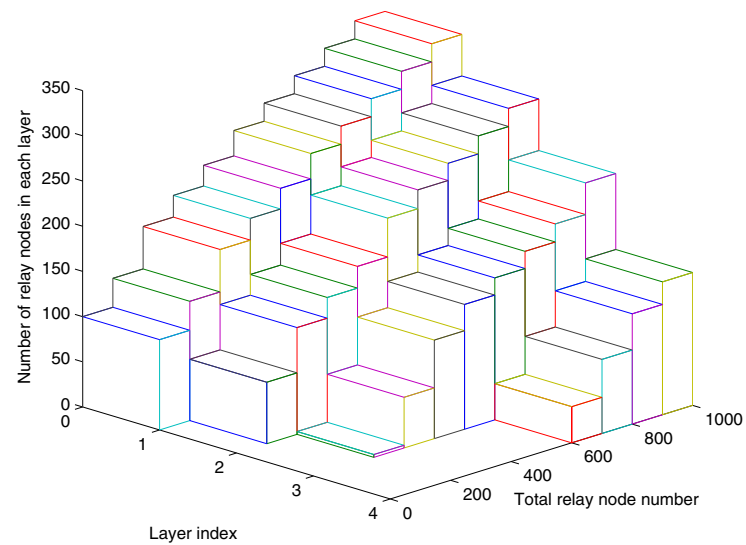

Fig. 2. The relay nodes distribution in different layers

be achieved even when only a small number of relays are introduced. For example, when only $10 \%$ of extra relay nodes are deployed, the network lifetime can be further extended more than $50 \%$ by applying the greedy heuristic deployment method. We can also see that if we deploy nodes randomly, the gain is very minor. For example, adding 1000 relay nodes can only increase the network lifetime by $50 \%$ if we deploy them randomly. On the other hand, the network lifetime can be tripled if the 1000 relays are deployed in an efficient way, as demonstrated by the figure.

Fig. 1(b) shows the results for a smaller network size. Now we set the network radius to be $5 d_{\text {char }}$, that is, there are 5 layers in network. From these results we can see that significant network lifetime extension still can be achieved by joint relay deployment and power control. For example, when only $10 \%$ of extra relays are added, the network lifetime can be extended by more than $30 \%$. If we compare the results in Fig. 1(a) and Fig. 1(b), we can see that more network extension can be achieved in a larger network, where $10 \%$ extra relays can bring $50 \%$ lifetime extension. This is because in larger network, the bottleneck effect around the sink is more significant. This also suggests that the proposed scheme can effectively alleviate the bottleneck effect.

To help better understand the results, we have plotted the relay deployment obtained by the proposed greedy heuristic for the 10-layer network case. The results are illustrated in Fig. 2. Given the total number of relay nodes, which is denoted by the total relay node number, this figure plots the number of relay nodes deployed in each layer. For example, when 300 relays will be added, 179 of them will be put into the first (innermost) layer, 


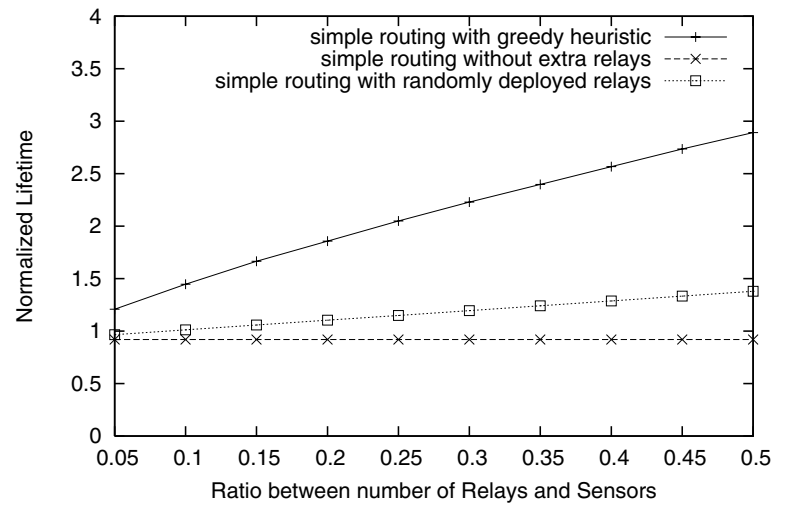

Fig. 3. Normalized network lifetime by deploying extra relays using simple routing

118 of them will be put into the second layer, and 3 of them will be put into the third layer. From these results we can see that most relay nodes will be deployed in the inner several layers. For example, even when the number of relays is 1000 , there are still no relay nodes that will be deployed to the layers beyond the 4th layer.

One problem with the proposed joint node deployment and adaptive data propagation scheme is that it involves a complicated routing scheme. The traffic from one layer may need to be transmitted to several different layers due to the optimality requirement. In our previous work [7], we proposed one simple traffic split and power control algorithm: all traffic will be sent to the next inner layer or the sink directly, which is shown to be optimal in some cases and near optimal in the other cases. To make the proposed method more practical, from now on when we do adaptive data propagation, we will adopt this simple strategy. To reflect this change, we modify the original optimization by adding one more constraint: $\left\{x_{i, j}=0, j \neq 0 \& j \neq i-1\right\}$. Then we can apply the proposed greedy heuristic to re-solve the joint optimization problem.

Fig. 3 shows the results for 10-layer network case after applying the modified greedy heuristic. First, from these results we can see that significant gain can still be achieved by the modified scheme, though the modified scheme is much simpler than the original scheme. For example, when adding $10 \%$ extra relay nodes, the network lifetime can still be extended by around $50 \%$. Second, comparing the results in Fig. 3 and Fig. 1(a), we can see that the modified scheme causes slight performance loss compared with the original case. For example, when 1000 relays are deployed in the network, the network

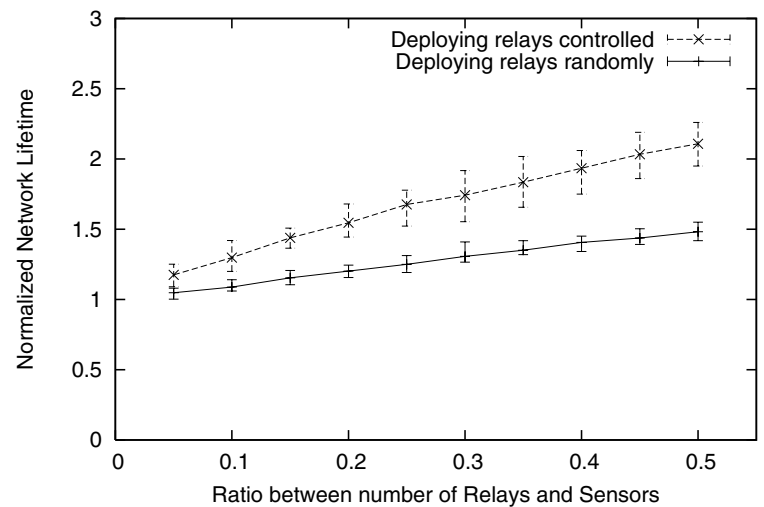

Fig. 4. Normalized network lifetime by deploying extra relays using simple routing

lifetime is extended by $190 \%$ instead of $200 \%$. Since the loss is very small, we believe the modified scheme should be adopted when doing joint node deployment and adaptive data propagation due to its simplicity.

\section{Simulation}

This section evaluates the performance of proposed greedy heuristic in randomly deployed sensor network.

The simulation is set up on a randomly deployed circular sensor network with radius $R=10 d_{\text {char }}$. The sink is located at the center of the area. For each node (sensor or relay), we set $\gamma_{1}=45 \mathrm{~nJ} / \mathrm{bit}, \gamma_{2}=135 \mathrm{~nJ} / \mathrm{bit}$, $\beta=10 \mathrm{pJ} / \mathrm{bit} / \mathrm{m}^{2}$ for $\alpha=2$, therefore $d_{\text {char }} \simeq 134 \mathrm{~m}$. The initial energy is 2000 Joule per node. In each unit time (round) each sensor generates a 25-Byte message to be sent to the sink, and relays generate none.

The simulation results are the average from 10 different randomly generated networks. The baseline lifetime is achieved by using simple adaptive data propagation scheme [7] without extra relays. It is worth pointing out that in our simulation, interference has not been considered separately.

In each test, we first randomly deploy the sensor nodes into the network according to the uniform distribution with density $\frac{20}{\pi d_{c h a r}^{2}}$. We then randomly deploy the relays in layers according to the numerical results from Section III. During test, each node transmits its traffic either to the sink or its neighbors which are around $d_{c h a r}$ away [7].

The simulation results are illustrated in Fig. 4. From the results we can see that the proposed scheme brings 
considerable performance gain. For example, when $10 \%$ relay nodes are added into the network strategically, around $35 \%$ lifetime extension can be obtained. The results also show the same trend as the numerical results (Fig. 3), but with lower lifetime extension. This is because when we model the problem into the joint optimization problem (3)-(8), we make an approximation that adding relay nodes into the network is equivalent to adding energy to the sensor nodes. In real network, when a sensor runs out of energy, the network will lose the coverage and then the network will terminate; however, the relay nodes may still have considerable energy left. In this situation, adding relay nodes into the network is actually not equivalent to adding energy to the sensor nodes. So this approximation leads to the gap between numerical results and simulation results.

\section{CONCLUSiON}

Battery powered wireless sensor network is extremely energy constrained. The all-to-one communication pattern in general homogeneous sensor networks makes the sensors around the sink deplete the energy much faster than faraway sensors due to the heavily relay burden. To conquer this problem, various schemes are proposed. In this paper we solve this problem by joint relay deployment and adaptive data propagation scheme. We model the problem as a mixed-integer nonlinear programming problem, which is known to be NP-hard. We then solve the optimization problem using greedy heuristic which is verified to be effective by both numerical results and simulation results.

\section{REFERENCES}

[1] C. Perkins, Ad Hoc Networking, Addison-Wesley, 2000.

[2] C. K. Toh, Ad Hoc Mobile Wireless Networks: Protocols and Systems, Prentice Hall PTR, 2001.

[3] J. Luo and J.-P. Hubaux, "Joint mobility and routing for lifetime elongation in wireless sensor networks," in IEEE INFOCOM, 2005.

[4] X. Liu and P. Mohapatra, "On the deployment of wireless sensornetworks," in the Third International Workshop on Measurement, Modeling, and Performance Analysis of Wireless Sensor Networks (SenMetrics), 2005.

[5] Y. T. Hou, Y. Shi, H. D. Sherali, and S. F. Midkiff, "Prolonging sensor network lifetime with energy provisioning and relay node placement," in IEEE SECON, September 2005.

[6] Y.Xin, T. Guven, and M.A. Shayman, "Relay deployment and power control for lifetime elongation in sensor networks," in IEEE ICC, June 2006.
[7] F. Sun and M. Shayman, "Lifetime maximizing adaptive traffic distribution and power control in wireless sensor network," Tech. Rep., University of Maryland, The Institute for Systems Research, 2006.

[8] S. Meguerdichian, F. Koushanfar, M. Potkonjak, and M.B. Srivastava, "Coverage problems in wireless ad-hoc sensor networks," in IEEE Infocom, April 2001, vol. 3, pp. 13801387.

[9] H. Zhang and J. C. Hou, "On the upper bound of a-lifetime for large sensor networks," ACM Transactions on Sensor Networks, vol. 1, no. 2, pp. 272-300, November 2005.

[10] S. Vasudevan, C. Zhang, D. Goeckel, and D. Towsley, "Optimal power allocation in wireless networks with transmitter-receiver power tradeoffs," in Proceedings of IEEE INFOCOM, April 2006.

[11] M. Bhardwaj and A. P. Chandrakasan, "Bounding the lifetime of sensor networks via optimal role assignments," in IEEE Infocom, March 2002.

[12] W. Heinzelman, Application-Specific Protocol Architectures for Wireless Networks, Ph.D. thesis, Massachusetts Institute of Technology, 2000.

[13] M. Bhardwaj and A. P. Chandrakasan, "Bounding the lifetime of sensor networks via optimal role assignments," in IEEE Infocom, March 2002.

[14] Christian Bettstetter, "On the minimum node degree and connectivity of a wireless multihop network," in Proceedings of the 3rd ACM MOBIHOC, 2002. 